\title{
Misleading findings of homozygosity mapping resulting from three novel mutations in NPHS1 encoding nephrin in a highly inbred community
}

Yaacov Frishberg, $M D^{1}$, Ziva Ben-Neriah, $M D^{2}$, Maija Suvanto, $B S c^{3}$, Choni Rinat, $M D^{1}$, Minna Männikkö, $P h D^{4}$, Sofia Feinstein, $M D^{1}$, Rachel Becker-Cohen, $M D^{1}$, Hannu Jalanko, $M D, P h D^{3}$, Joel Zlotogora, $M D, P h D^{5}$, and Marjo Kestilü, $P h D^{6}$

\begin{abstract}
Purpose: Congenital nephrotic syndrome of the Finnish type (CNF, NPHS1) is a rare autosomal recessive disease caused by mutations in the NPHS1 gene encoding nephrin. We diagnosed congenital nephrotic syndrome in 12 children living in a village near Jerusalem. Most of the inhabitants are descendants of one Muslim family and have maintained their isolation by preference of consanguineous marriages. The aim of this study was to confirm that the NPHS1 gene is responsible for congenital nephrotic syndrome in our population, applying homozygosity mapping. Methods: DNA samples were genotyped by four microsatellite markers that were in linkage disequilibrium with the NPHS1 gene on chromosome 19q13.1. Immunoperoxidase staining was used to study the expression of nephrin, and mutations were subsequently identified by direct sequencing of the entire coding region of the NPHS1 gene. Results: Haplotype analysis revealed several different haplotypes, leading us to assume erroneously that there was genetic heterogeneity of congenital nephrotic syndrome. Because nephrin was completely absent in kidney tissue of one patient, direct sequencing of all DNA samples was performed, yielding three novel mutations: c.1138C > T (p.Gln380X), c.2160_ 2161insC (p.Cys721fs), and c.1707C >G (p.Ser569Arg). Patients were either homozygous for one of these mutations or compound heterozygotes, and they differed in their phenotype. Conclusion: We report the potential pitfalls of performing homozygosity mapping in a highly consanguineous population and discuss the phenomenon of multiple mutations in a given gene within an isolate. Genet Med 2007:9(3):180-184.
\end{abstract}

Key Words: homozygosity mapping, consanguineous populations, congenital nephrotic syndrome

\section{INTRODUCTION}

Congenital nephrotic syndrome of the Finnish type (CNF; NPHS1, MIM 256300) is an autosomal recessively inherited kidney disease. This disorder is relatively prevalent in Finland, where the incidence is approximately 1 in 8200 live births. ${ }^{1}$ It has been diagnosed worldwide, although with lower frequency. The highest incidence has been reported among the Old Order Mennonites in Lancaster County, Pennsylvania, reaching 1 in 500 live births. ${ }^{2}$ Affected children are often born prematurely with an enlarged placenta weighing more than $25 \%$ of the baby's birth

From the ${ }^{1}$ Division of Pediatric Nephrology, Shaare Zedek Medical Center, Jerusalem, Israel, ${ }^{2}$ Department of Human Genetics, Hadassah University Medical Center, Jerusalem, Israel; ${ }^{3}$ Hospital for Children and Adolescents, University of Helsinki, Finland; ${ }^{4}$ Department of Medical Biochemistry and Molecular Biology, University of Oulu, Oulu, Finland; ${ }^{5}$ Department of Community Genetics, Public Health Services, Ministry of Health, Jerusalem, Israel; ${ }^{6}$ Department of Molecular Medicine, National Public Health Institute, Helsinki, Finland.

Yaacov Frishberg, MD, Division of Pediatric Nephrology, Shaare Zedek Medical Center, PO Box 3235, Jerusalem 91031, Israel. E-mail: yaacov@md.huji.ac.il

The authors declare no conflict of interest.

Submitted for publication September 3, 2006.

Accepted for publication December 14, 2006.

DOI: 10.1097/GIM.0b013e318031c7de weight. Nephrotic syndrome (NS) is detected before the age of 3 months but usually within the first week of life. NS manifests with massive proteinuria resulting in severe hypoalbuminemia and generalized edema. Other signs include abdominal distention, umbilical hernias, and widened sutures and fontanelles. Kidney transplantation is the only definitive treatment for CNF, and it is usually performed at the age of 1 to 2 years. ${ }^{3}$

Mutations in the NPHS1 gene, encoding nephrin, were found to be responsible for CNF. 4 Thus, more than 80 different mutations have been described, making prenatal DNA diagnosis possible in families with known disease-causing mutations. Nephrin is expressed in the slit diaphragm joining the podocyte foot processes and is the critical component in the glomerular filtration barrier. $^{5}$

In this study, we identified three novel NPHS1 mutations in a highly inbred community.

\section{PATIENTS AND METHODS}

\section{Patients}

This cohort consisted of 12 affected patients: two pregnancies had been terminated, three infants died as a result of CNF during early infancy, and the only two children who survived 
had dissimilar phenotype. Another five infants diagnosed with CNF died shortly after birth and before this study. Their DNA samples were unavailable. All the nuclear families live in a village near Jerusalem, in which most of the inhabitants are descendants of a single Arab-Muslim family who settled in this site approximately 250 years ago. The village kept its genetic isolation by preference of consanguineous marriages. Demographic and genetic data on individuals born in the village were collected through interviews and entered into a computer database (Reunion, Leister Production Inc., Mechanicsburg, PA). The data included had been verified by interviews of many of the village elders. As not all family connections have been elucidated, it was only possible to define the relationships to an extent that enabled us to cluster all the individuals involved into two pedigrees. These two pedigrees are clearly also related to each other in a way that has not yet been disclosed, and for that reason, Figure 1 certainly underestimates the complexity of consanguinity in this isolate. Several autosomal recessive diseases, including cystic fibrosis and primary hyperoxaluria type 1 , are also prevalent in this village. ${ }^{6,7}$

The study was approved by the Ethical Committee of the Shaare Zedek Medical Center in Jerusalem, Israel.

\section{METHODS}

\section{Marker genotyping}

Genomic DNA was isolated according to standard laboratory protocol from fresh peripheral blood samples or amniotic fluid, using conventional molecular biology techniques. Seventeen DNA samples were genotyped by D19S610, D19S608, D19S224, and D19S220 microsatellite markers (www.gdb. org). Genotypes were not obtained for two individuals (IV:5 and V:8, Fig. 1, Pedigree A). One primer in each polymerase chain reaction (PCR) reaction was end-labeled with ${ }^{32} \mathrm{P}$, and the PCR products were run on polyacrylamide gel electrophoresis. After gels were autoradiographed overnight on x-ray films, alleles were scored visually.

\section{Sequencing}

All 29 exons of the NPHS1 gene were amplified by PCR with flanking intronic primers, and the reactions were performed in total volumes of $25 \mu \mathrm{l}$ as described previously. ${ }^{8}$ Exons 12 to 13 required a denaturation temperature of $98^{\circ} \mathrm{C}$ and the addition of betaine to the reaction mixture. PCR products were enzymatically purified using shrimp alkaline phosphatase and Exonuclease I (Amersham Pharmacia) and bi-directionally sequenced with BigDye Terminator chemistry (v.3.1) on 3730 DNA Analyzer (Applied Biosystems). Sequences including intron-exon splice sites were analyzed by GeneComposer version 1.1.0.1051.

\section{Nephrin expression}

Immunoperoxidase staining was performed according to Patrakka et al. ${ }^{9}$ In this study, renal samples from patient IV:13 (Fig. 1, Pedigree B) and a healthy control were stained using polyclonal antibody directed against the intracellular part of nephrin.

\section{RESULTS}

When mapping of the CNF-causing gene (NPHS1) was first reported, we attempted to determine the pathogenic role of this gene in our patients with CNF. This was done by looking for homozygosity in the region delineated in Finland. The markers used for homozygosity mapping were D19S610, D19S608, D19S224, and D19S220, which are known to be in linkage disequilibrium with the NPHS1 gene. ${ }^{4}$ All markers were located distally to the NPHS1 gene with the following distances: NPHS1 - $3.7 \mathrm{~kb}$ - D19S610 - $117 \mathrm{~kb}$ - D19S608 - 64 $\mathrm{kb}$ - D19S224 - 1.9 Mb - D19S220. The analyzed markers did not flank the NPHS1 gene, but D19S610 locates very close to the 5'end of NPHS1, and recombinations were never observed between NPHS1 and D19S610 when these markers were analyzed in the Finnish patients with $\mathrm{CNF}^{4}$ and patients with Polycystic Lipomembranous Osteodysplasia with Sclerosing Leukoencephalopathy (PLOSL). ${ }^{10}$ Because we assumed one single mutation was the cause of CNF in this highly inbred community, the different haplotypes observed led us to conclude that the disease-causative gene in our patients is mapped to a different locus. The observed haplotypes are shown in Figure 1.

Later, when another affected child was born in the village (IV:13 in Fig. 1, Pedigree B), the NPHS1 antibody staining was available, and it demonstrated that nephrin expression was completely absent in his kidney biopsy (Fig. 2). This finding directed us to perform sequencing of the entire coding region of NPHS1 in the DNA samples of patients and their parents. This analysis showed that there are three novel mutations in this cohort: c.1138C > T, c.2160_2161insC, and c.1707C > G. All three mutations segregate with the disease in these families. Patients were found to be either homozygous or compound heterozygotes (Fig. 1). The c.2160_2161insC mutation leads to a frameshift at cysteine 721 resulting in a stop codon at amino acid 725 (Cys721fs). The c.1138C > T mutation causes a stop codon at amino acid 380 (p.Gln380X), also leading to truncation of the protein. The third mutation is c.1707C $>$ G, resulting in the substitution of serine to arginine in position 569 (p.Ser569Arg). This mutation was found in the affected fetus IV:4 and his mother III:3 (Fig. 1, Pedigree B). No further changes were observed when all the exons and intron-exons boundaries were sequenced, suggesting that Ser569Arg is an additional disease-causing mutation in this family. Although the PolyPhen analysis (coot.embl.de/PolyPhen/) predicted that this change might be rather benign, patient IV:3 (Fig. 1, Pedigree B) died as a result of typical CNF, suggesting that nephrin function was deranged. Unfortunately, we could not analyze the DNA of patient IV:3, but it can be assumed that she carried the same mutations as the affected fetus (IV:4) in the very same family. Because serine is a small hydrophilic polar amino acid, whereas arginine is a much bigger hydrophilic base, it is possible that the abnormal function of nephrin in this case may be attributable to the size difference, leading to an 
A
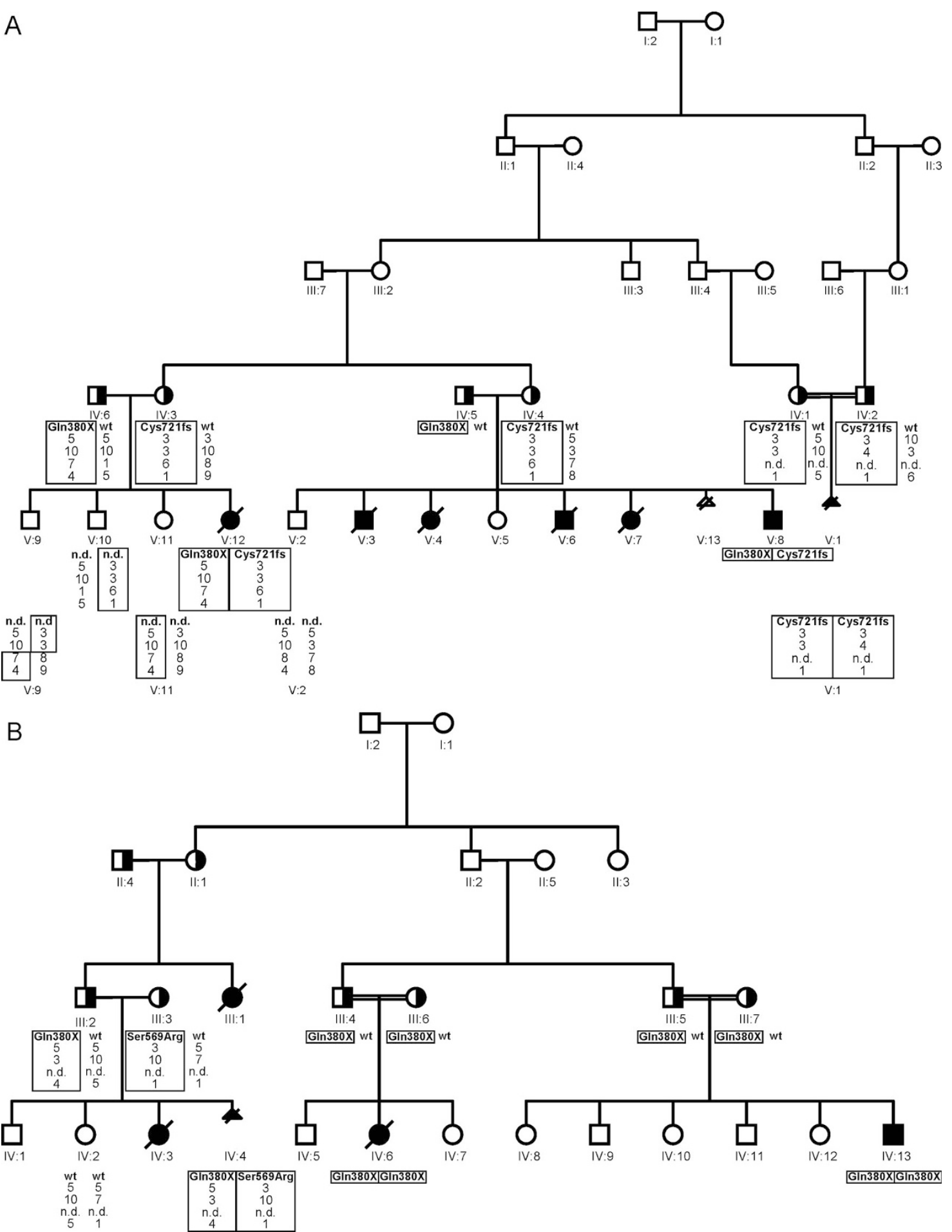

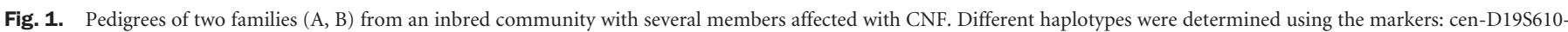

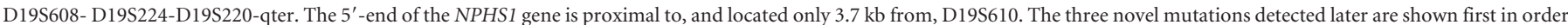
within the haplotype. The carrier state of patients' siblings is not marked as the mutation analyses have not included these individuals. n.d., not determined.

aberrant folding of nephrin. Furthermore, the fact that serine 569 is conserved among species points to the important role that this amino acid plays in nephrin function. This mutation was not detected in 40 healthy unrelated individuals from the same ethnic background. The p.Ser569Arg mutation was only recently introduced into this isolate, with the marriage of a female carrier from Jerusalem to a man from the village. The other two mutations have been present in the village for a longer period of time and are widely spread among the inhabitants. Analysis of the family data suggests that both mutations occurred in two branches of the pedigrees at least six generations (120 years) ago. 


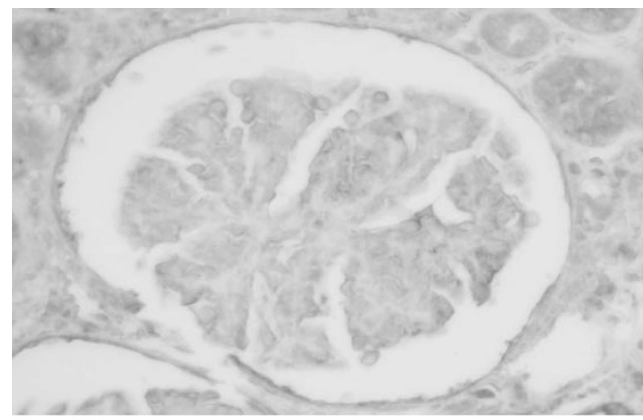

A

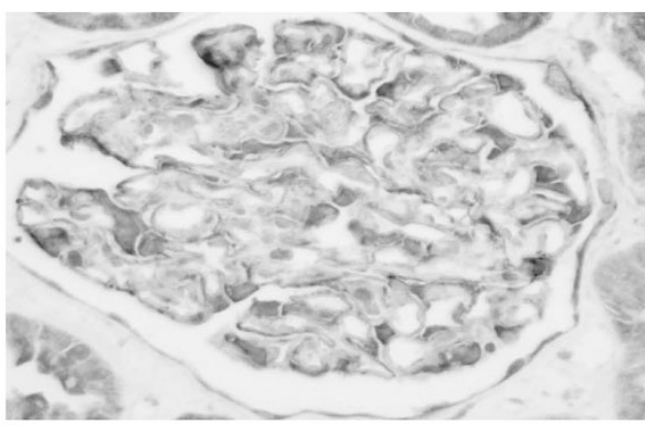

B

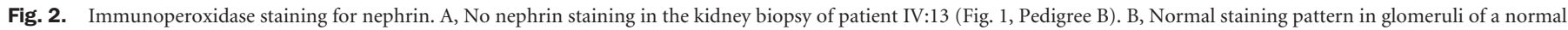
individual. Original magnification $\times 200$.

When comparing the observed haplotypes with the identified mutations, it can be concluded that the haplotypes 3-3-6-1 and 3-4-nd-1 represented the Cys721fs mutation. Allele 4 of D19S608 most probably results from allele mutation, although double recombination cannot be excluded, even if recombinations in this region are very infrequent. Two haplotypes, 5-107-4 and 5-3-nd-4, stood for the Gln380X mutation, and the difference in the haplotypes is the result of historical recombinations in an original haplotype. The haplotype 3-10-nd-1 denoted the Ser569Arg mutation.

Variability in phenotype was encountered in two affected individuals who survived. The remaining five affected patients included two pregnancies that had been terminated and three babies who died during early infancy. The p.Gln380X homozygote (IV:13 in Fig. 1, Pedigree B) required daily albumin infusion until he was 2 years old, when a bilateral nephrectomy was performed, followed by a successful living donor renal transplantation. He has not experienced post-transplantation nephrotic syndrome. The compound heterozygote [(p.Gln380X/ p.Cys721fs) (V:8 in Fig. 1, Pedigree A)] was weaned from albumin infusions at the age of 18 months. His renal function gradually deteriorated until he reached end-stage renal failure at the age of 3.8 years. He has been treated with chronic hemodialysis and is awaiting kidney transplantation. Neither of the patients responded with decreasing proteinuria to treatment with ACE inhibitors.

After the detection of these three mutations in NPHS1, we initiated targeted screening in the village. Our preliminary results show that, of the 116 individuals analyzed, 13 are carriers of the p.Cys721fs mutation, and one was found to carry the p.Gln380X mutation.

\section{DISCUSSION}

Homozygosity mapping provides a rapid and efficient means of mapping autosomal recessive genes in consanguineous families and genetically isolated populations by identifying chromosomal regions that show homozygous identity-by-descent segments in numerous samples. ${ }^{11}$ Several mechanisms may have accounted for loss of homozygosity in an inbred isolate like that reported herein, which could lead to the false conclusion that the studied disorder is not mapped to a known genetic locus. The first mechanism involves the occurrence of allelic heterogeneity within a consanguineous community and would result in loss of homozygosity in flanking markers and failure to detect linkage. In this study, we found three novel mutations in the NPHS1 gene encoding nephrin and causing CNF in a highly inbred Arab village. Because of the different mutations, several haplotypes were observed in the initial haplotype analysis, resulting in the erroneous conclusion that there was genetic heterogeneity in recessive congenital nephrotic syndrome. Similar examples of multiple rare mutations in an inbred population, which resulted in loss of homozygosity, were described among the Amish community involving the thyroid peroxidase gene as well as nephrin. ${ }^{2,12}$ It was also noted in consanguineous families from North America with the enhanced S-cone syndrome. ${ }^{13}$ Another option is genetic heterogeneity when several different genes are responsible for an identical phenotype. CNF is a heterogeneous group of clinical entities: some are of genetic etiology and others are not. There are at least two distinct recessive disorders that may present with CNF: mutations in NPHS1 encoding nephrin or NPHS2 encoding podocin. ${ }^{14,15}$ There may even be a di-genic inheritance of NPHS1 and NPHS2 mutations that result in a "tri-allelic" disorder, in which an infant is homozygous for a mutation in one gene and carries a mutation in the other. ${ }^{16}$ Analyzing DNA samples from a phenotypically identical but genetically heterogeneous group of patients would have resulted in failure of homozygosity mapping. The third proposed explanatory mechanism that we initially considered is that DNA samples from fetuses diagnosed based on increased $\alpha$-fetoprotein were analyzed together with those of live-born affected individuals. Several years after the cloning of nephrin, it was discovered that fetuses that are heterozygotes for a mutation in NPHS1 have abnormal renal histological findings and increased $\alpha$-fetoprotein concentrations in maternal serum and amniotic fluid that are indistinguishable from truly affected individuals. ${ }^{17}$ If some of the patients diagnosed prenatally were in fact carriers of a mutation in NPHS1, it would explain that they were not homozygous in the first mapping. This possibility was later ruled out because the affected fetuses were indeed either homozygous for one of the mutations or compound heterozygotes. 
The infant who was a compound heterozygote for the mutations (p.Gln380X/p.Cys721fs; V:8 in Fig. 1, Pedigree A) had a milder disease course compared with the homozygote for the mutation (p.Gln380X, IV:13 in Fig. 1, Pedigree B). It is unclear whether phenotypic variability is attributable to the causative mutation. The fact that so-called monogenic diseases may not show consistent relationships between mutant genotype and phenotype is well established. ${ }^{18,19}$ In Malta, there is wide phenotypic variability in CNF, ranging from patients who die of the disease in infancy to children (often girls) with mild proteinuria at the age of 19 years, all of whom are homozygous for the p.Arg1160X founder mutation in NPHS1. ${ }^{16}$ None of the carriers in our cohort had proteinuria or compromised renal function.

In this article, we described an Arab village that has kept its genetic isolation for approximately 250 years by preference of consanguineous marriages. One would expect a single founder mutation to be responsible for the high frequency of the rare, recessive CNF. Instead, three novel mutations in NPHS1 were detected. The phenomenon of multiple mutations in rare recessive disorders among the Arab population in Israel has been previously noted. ${ }^{7,20,21}$ Several possible explanations have been proposed to delineate the responsible mechanism. Spontaneous mutations occur constantly in all genes, but they are only detected through the change in phenotype they cause. Theoretically, even if the de novo mutation rate is normal, a new deleterious recessive allele will likely become homozygous because of the high rate of consanguinity. Alternatively, there may be a higher mutation rate, which will obviously lead to a significant number of affected individuals. Finally, there may be a yet unidentified advantage to the heterozygote that is combined with the unique structure of the community involving high consanguinity and large families. The possibility that either one of the two more common mutations in nephrin was introduced into the village from another isolate cannot be excluded. In fact, the rarest of the three mutations originated from an unrelated family from Jerusalem. The mutations we describe are novel and have not been reported in other Arab communities in Israel or in neighboring countries.

\section{References}

1. Patrakka J, Kestila M, Wartiovaara J, Ruotsalainen V, et al. Congenital nephrotic syndrome (NPHS1): features resulting from different mutations in Finnish patients. Kidney Int 2000;58:972-980.
2. Bolk S, Puffenberger EG, Hudson J, Morton DH, et al. Elevated frequency and allelic heterogeneity of congenital nephrotic syndrome, Finnish type, in the Old Order Mennonites. Am J Hum Genet 1999;65:1785-1790.

3. Holmberg C, Tryggvason K, Kestilä M, Jalanko H. Congenital nephrotic syndrome. In: Avner EHW, Niaudet P, editors. Pediatric nephrology. Baltimore: Lippincott Williams \& Wilkins, 2004:503-516.

4. Kestilä M, Lenkkeri U, Männikkö M, Lamerdin J, et al. Positionally cloned gene for a novel glomerular protein-nephrin-is mutated in congenital nephrotic syndrome. Mol Cell 1998;1:575-582.

5. Ruotsalainen V, Patrakka J, Tissari P, Reponen P, et al. Role of nephrin in cell junction formation in human nephrogenesis. Am J Pathol 2000;157:1905-1916.

6. Chiba-Falek O, Nissim-Rafinia M, Argaman Z, Genem A, et al. Screening of CFTR mutations in an isolated population: identification of carriers and patients. Eur J Hum Genet 1998;6:181-184.

7. Rinat C, Wanders RJ, Drukker A, Halle D, et al. Primary hyperoxaluria type I: a model for multiple new mutations in a monogenic disease within a distinct ethnic group. J Am Soc Nephrol 1999;10:2352-2358.

8. Lenkkeri U, Männikkö M, Mccready P, Lamerdin J, et al. Structure of the gene for congenital nephrotic syndrome of the finnish type (NPHS1) and characterization of mutations. Am J Hum Genet 1999;64:51-61.

9. Patrakka J, Ruotsalainen V, Ketola I, Holmberg C, et al. Expression of nephrin in pediatric kidney diseases. J Am Soc Nephrol 2001;12:289-296.

10. Pekkarinen P, Kestila M, Paloneva J, Terwillign J, et al. Fine-scale mapping of a novel dementia gene, PLOSL, by linkage disequilibrium. Genomics 1998;54:307-315.

11. Sheffield VC, Nishimura DY, Stone EM. Novel approaches to linkage mapping. Curr Opin Genet Dev 1995;5:335-341.

12. Pannain S, Weiss RE, Jackson CE, Dian D, et al. Two different mutations in the thyroid peroxidase gene of a large inbred Amish kindred: power and limits of homozygosity mapping. J Clin Endocrinol Metab 1999;84:1061-1071.

13. Miano MG, Jacobson SG, Carothers A, Hanson I, et al. Pitfalls in homozygosity mapping. Am J Hum Genet 2000;67:1348-1351.

14. Boute N, Gribouval O, Roselli S, Benessy F, et al. NPHS2, encoding the glomerular protein podocin, is mutated in autosomal recessive steroid-resistant nephrotic syndrome. Nat Genet 2000;24:349-354.

15. Frishberg Y, Rinat C, Megged O, Shapira E, et al. Mutations in NPHS2 encoding podocin are a prevalent cause of steroid-resistant nephrotic syndrome among Israeli-Arab children. J Am Soc Nephrol 2002;13:400-405.

16. Koziell A, Grech V, Hussain S, Lee G, et al. Genotype/phenotype correlations of NPHS1 and NPHS2 mutations in nephrotic syndrome advocate a functional interrelationship in glomerular filtration. Hum Mol Genet 2002;11:379-388.

17. Patrakka J, Martin P, Salonen R, Kestilä M, et al. Proteinuria and prenatal diagnosis of congenital nephrosis in fetal carriers of nephrin gene mutations. Lancet 2002;359: 1575-1577.

18. Dipple KM, McCabe ER. Phenotypes of patients with "simple" Mendelian disorders are complex traits: thresholds, modifiers, and systems dynamics. Am J Hum Genet 2000;66:1729-1735.

19. Scriver CR. Why mutation analysis does not always predict clinical consequences: explanations in the era of genomics. J Pediatr 2002;140:502-506.

20. Zlotogora J, Gieselman V, Bach G. Multiple mutations in a small geographic area: a common phenomenon? Am J Hum Genet 1996;64:83-88.

21. Zlotogora J, Carasquillo M, Barges S, Shalev SA, et al. High incidence of deafness from three frequent connexin 26 mutations in an isolated community. Genet Test 2006;10:40-43. 